Brit. F. vener. Dis. (1976) $5 \frac{2}{\phi}, 224$

\title{
Reactivity of lymphocytes from patients with syphilis towards T. pallidum antigen in the leucocyte migration and lymphocyte transformation tests
}

\author{
E. FROM, K. THESTRUP-PEDERSEN, AND H. THULIN \\ From the Department of Dermatology and Venereology, Marselisborg Hospital, and the Institute of \\ Medical Microbiology, University of Aarhus, Denmark
}

\section{Summary}

The reactivity of lymphocytes to Treponema pallidum antigen was studied before and after treatment in nine patients with early syphilis using a leucocyte migration test and a lymphocyte transformation test. Lymphocyte reactivity was also investigated in six patients treated for syphilis within the last 4 years, and in five untreated patients with a positive result to the $T$. pallidum immobilization test, but negative results to other serum tests for syphilis antibodies and without any known exposure to risk of infection by syphilis. Ten seronegative patients with different dermatological disorders served as a control group.

A significant increase in lymphocyte reactivity to $T$. pallidum antigen was recorded in both tests in vitro after treatment. There was no difference in lymphocyte reactivity to $T$. pallidum antigen between the other patients studied and the control group.

In early syphilis the spontaneous migration was found to be inhibited before treatment. Tuberculin skin tests were also performed and found to be suppressed in patients with primary and secondary syphilis.

No difference in phytohaemagglutinin response was found between any of the groups. Plasma from patients with primary and secondary syphilis was found to change the in vitro reactivity of normal lymphocytes when stimulated with different mitogens.

\section{Introduction}

Syphilis induces a distinct humoral immune reponse in patients, and antibodies develop readily but do

Received for publication June 30, 1975

Address for reprints: Dr E. From, Marselisborg Hospital, Skanderborgvej 9, 8000 Aarhus C, Denmark not seem to be related to the termination of the disease. It has been suggested that a cell-mediated immune reaction against $T$. pallidum may be important (Fulford and Brostoff, 1972; Wright and Grimble, 1974).

We have studied humoral and cellular immune reactions in patients with early syphilis before and after treatment. Investigations for immunosuppressive factors in plasma were made using the lymphocyte transformation test.

\section{Patients and methods}

Twenty patients (sixteen men and four women) were investigated. They were divided into three groups. (1) Nine patients with early syphilis, four in the primary and five in the secondary stage. They all had positive serological standard tests for syphilis (STS) including Wassermann (WR), Kahn (KR), and Meinicke (MR); the $T$. pallidum immobilization test (TPI) was reactive in eight patients. When the diagnosis of syphilis was established, blood was taken for the immunological investigations before the patients were treated with procaine penicillin Retard ${ }^{\circledR}$ (PAM) 600,000 i.u. daily for 10 days. One month and 2 to 6 months after treatment they were again examined and blood was taken for repeated immunological investigations.

(2) Six patients who had been treated for syphilis within the last 4 years. All still showed a positive TPI; STS were positive in four patients and negative in two.

(3) Five patients having a positive TPI, but without known exposure to syphilis and with negative results to STS.

Ten patients with different dermatological disorders, matched by age and with negative STS and TPI results, served as a control group.

In vitro cultures from patients and controls were performed in parallel.

Immunological investigations

Tuberculin skin testing was done twice in seven of nine patients in Group 1. Peripheral blood lymphocytes were studied in vitro for reactivity towards a $T$. pallidum antigen using leucocyte migration and lymphocyte 
transformation tests. Plasma samples were taken before and after treatment of six patients in Group 1 and investigated for the existence of immunosuppressive factors.

\begin{abstract}
Skin testing
Skin reactivity was assessed by means of a tuberculin skin test (Mantoux test) in seven of nine patients in Group 1 before treatment and repeated after 1 month. One tuberculin unit (1 T.U.) of purified protein derivative (PPD) was injected intradermally into the dorsal side of the forearm in a volume of $0.1 \mathrm{ml}$. The reading was done after $72 \mathrm{hrs}$ by drawing the area of induration on a transparent foil placed over the forearm. The areas were measured using an electronic planometer (HewlettPackard Digitizer, Model 9107A) and expressed in $\mathrm{cm}^{2}$. In five cases the second test was performed in the other forearm. Seven normal persons, not identical with our control group, were also skin-tested with PPD in a similar way. This was done to see if the size of a second skin test could be influenced by the primary test. In these persons the second skin test was always given in the opposite forearm.
\end{abstract}

\section{T. pallidum antigen}

The antigen (TP-antigen) was supplied and prepared by Treponematoseafdelingen, Statens Seruminstitut, Copenhagen. The treponemes (Nichols strain) were obtained from rabbits, which had received intratesticular injection of $2 \times 10^{7}$ micro-organisms 12 to 16 days earlier. The inflamed testes were removed aseptically, minced with scissors, and shaken in sterile isotonic saline for a short time. Gross cellular debris was removed by centrifugation at $2,000 \mathrm{rpm}$ for $10 \mathrm{~min}$. and the supernatant was subjected to centrifugation at $13,000 \mathrm{rpm}$ for $30 \mathrm{~min}$. The sediment was re-suspended in sterile isotonic saline to give a concentration between $1 \times 10^{8}$ and $3 \times 10^{8}$ treponemes per $\mathrm{ml}$. The number of treponemes per $\mathrm{ml}$ was established by calibrated darkfield microscopy. The organisms were subjected to ultrasonification for $90 \mathrm{sec}$. at $0^{\circ} \mathrm{C}$., using a MSE $150 \mathrm{Watt}$ ultrasonic disintegrator. The sonicated treponemes were stored at $-20^{\circ} \mathrm{C}$. until they were used. Two batches of antigen were used. The same batch was employed in both the lymphocyte transformation test and the leucocyte migration test. No difference was found between the batches.

\section{Leucocyte migration test (LMT)}

This was performed as described by Clausen (1973), but instead of using the standard procedure, $\mathrm{CO}_{2} /$ bicarbonate buffered agarose medium was substituted by HEPESbuffered medium (Thulin, Thestrup-Pedersen, and Ellegaard, 1975).

Venous blood was collected in $10-\mathrm{ml}$. polystyrene tubes containing 250 i.u. heparin. After sedimentation at $37^{\circ} \mathrm{C}$ for one $\mathrm{hr}$, the leucocyte-rich plasma was transferred to thrombotest tubes and spun at $220 \mathrm{G}$ for $5 \mathrm{~min}$. The pellets were washed three times in Hanks's balanced salt solution. The leucocytes were re-suspended in TC-199 with 10 per cent. horse serum. The sonicated TP-antigen, dissolved in TC-199, was added to half of the leucocytes, and the same amount of TC-199 without antigen was added to the other half. The concentration of sonicated
TP-antigen in the culture chambers corresponded to $6 \times 10^{6}$ treponemes per $\mathrm{ml}$.

After incubation at $37^{\circ} \mathrm{C}$. for $30 \mathrm{~min}, 7 \mu$ of leucocyte suspension were placed in the wells in agarose plates. Six control cultures and six antigen-containing cultures were set up. All plates were incubated at $37^{\circ} \mathrm{C}$. in water saturated atmospheric air. After $24 \mathrm{hrs}$ the migration areas were measured by means of a projection microscope. Migration indices (MI) were calculated as the ratio between the average areas of TP-antigen containing cultures and the control cultures.

\section{Lymphocyte transformation test (LTT)}

Approximately $20 \mathrm{ml}$ venous blood was drawn into a test tube containing phenol-free heparin to produce a final concentration of 20 i.u. per ml. The mononuclear cells were separated using Isopaque-Ficoll (Böyum, 1968). They were washed twice in TC-199 with 5 per cent. inactivated pooled A-serum and heparin 20 i.u. per $\mathrm{ml}$. The A-serum was a pool from four normal blood donors with negative STS; this was used because of difficulty in obtaining AB-serum. The cells were suspended in TC-199 with 15 per cent. pooled, inactivated A-serum to give a final concentration of $0.5 \times 10^{6}$ lymphocytes per $\mathrm{ml}$. Portions of $2 \mathrm{ml}$. of this suspension were placed in culture tubes, $15 \times 150 \mathrm{~mm}$.

It was found in the LMT that a concentration of $6 \times 10^{6}$ per $\mathrm{ml}$ sonicated $T$. pallidum organisms was non-toxic to the lymphocytes in this test. The optimal concentration of antigen is generally lower in the LTT than in the LMT, and the TP-antigen was therefore used in concentrations of $2.5 \times 10^{5}, 5 \times 10^{5}$, and $1 \times 10^{6}$ organisms per ml culture. At least two different concentrations of antigen were used $\left(5 \times 10^{5}\right.$ and $\left.1 \times 10^{6}\right)$. The reactivity of lymphocytes after addition of phytohaemagglutinin (PHA, Wellcome MR 68), $1 \mu \mathrm{g}$ per $\mathrm{ml}$, was also studied. No addition was made to the control cultures. In a few patients we did not achieve enough lymphocytes to set up cultures with all concentrations of TP-antigen and PHA, and some cultures were discarded because of bacterial contamination during the culture period. The missing results are marked as 'n.d.' in Table II. Cultures were made in triplicate and incubated at $37^{\circ} \mathrm{C}$. The duration was 5 days for cultures stimulated with TP-antigen and 3 days for cultures stimulated with PHA. 24 hours before harvesting, $0.2 \mu \mathrm{Ci}$ of 2-C-14-thymidine, (Amersham, England, specific activity $>50 \mu \mathrm{Ci}$ per $\mathrm{mM}$ ) was added. The cells were harvested on Whatman glass-fibre filters $(\mathrm{GF} / 82)$ and washed in saline and alcohol, and incorporated labelled thymidine was counted in a TriCarb scintillation counter. The results are expressed either as disintegrations per minute (DPM) or as stimulation indices (S.I.)

\section{S.I. $=\frac{\text { counts per minute of antigen stimulated culture }}{\text { counts per minute of control culture }}$}

A positive response was defined as a S.I. $\geqslant 2 \cdot 0$. An S.I. between 2.0 and 3.0 was considered to be a weakly positive reaction, $\geqslant 3.0$ a strongly positive reaction. For convenience, only the highest S.I. obtained at one of the three (or two) concentrations of antigen is recorded in the Tables. A positive S.I. was not restricted to one concentration of antigen. 
Investigation of plasma for immunosuppressive factors Plasma samples, which could be withdrawn after separation of blood on Isopaque-Ficoll, were inactivated at $56^{\circ} \mathrm{C}$. for $30 \mathrm{~min}$ and kept at $-20^{\circ} \mathrm{C}$. until use. Lymphocytes from two normal donors were used in all experiments and prepared as described above. A total of $2 \times 10^{5}$ lymphocytes was cultured in $0.2 \mathrm{ml} \mathrm{TC}-199$ with 5 per cent. pooled A-serum from normal blood donors in microculture plates (Linbro Chemical Co., IS-MRC-96). The A-serum was added to inhibit the non-specific influence on the results when using different plasma samples (Mangi, Dwyer, and Kantor, 1974). $20 \mu \mathrm{l}$ of test plasma were added to give a final total concentration of about 15 per cent. serum/plasma in the culture. The use of 10 per cent. normal plasma and 5 per cent. pooled A-serum instead of 15 per cent. pooled A-serum has earlier been found not to change the in vitro reactivity of normal lymphocytes (Thestrup-Pedersen, Ellegaard, Thulin, and Zachariae, unpublished observations). The lymphocytes were stimulated with PHA, 2.5 $\mu \mathrm{g}$ per $\mathrm{ml}$, concanavalin A (Con A) $25 \mu \mathrm{g}$ per $\mathrm{ml}$, and poke-weed mitogen (PWM) $25 \mu \mathrm{l}$ per $\mathrm{ml}$; these were concentrations known to give an optimal response of normal lymphocytes in the present culture system. The culture period was 3 days for all mitogens, and radioactive thymidine $(0.02 \mu \mathrm{Ci}$ per culture) was added during the last 24 hrs.

The results obtained in the cultures with the patient's plasma are given as a percentage of the response of lymphocytes from the donor, when cultured in a mixture of 10 per cent. autologous inactivated plasma and 5 per cent. A-serum. The mean percentage from the results of the two donors was calculated.

\section{Results}

The results from skin-testing seven patients in Group I with PPD are shown in Table I. It appears that the areas of induration were larger after treatment than during the acute disease. The increase was found to be significant $(P<0.05$; Wilcoxon test of pair differences). These findings are opposite to the results in the control group in which the primary skin reactions were larger than the secondarily applied skin tests, but this difference was not significant.

TABLE I Areas of induration after tuberculin skin testing in patients with primary and secondary syphilis and normal persons. Interval between first and second Mantoux test was 1 month. Areas expressed in $\mathrm{cm}^{2}$.

\begin{tabular}{|c|c|c|c|c|}
\hline \multirow[b]{2}{*}{ Case No. } & \multicolumn{2}{|c|}{ Patients with syphilis, Group 1} & \multicolumn{2}{|l|}{ Controls } \\
\hline & 1. Mantoux & 2. Mantoux & 1. Mantoux & 2. Mantoux \\
\hline $\begin{array}{l}1 \\
2 \\
3 \\
4 \\
5 \\
6 \\
7\end{array}$ & $\begin{array}{l}0.7 \\
0.7 \\
0.9 \\
0.5 \\
0.6 \\
0.2 \\
0.2\end{array}$ & $\begin{array}{l}1 \cdot 1 \\
0.9 \\
0.8 \\
1.6 \\
4.2 \\
0.9 \\
1.5\end{array}$ & $\begin{array}{l}1.4 \\
1.4 \\
0.9 \\
1.0 \\
1.1 \\
1.5 \\
1.0\end{array}$ & $\begin{array}{l}1 \cdot 1 \\
1 \cdot 0 \\
0 \cdot 8 \\
0 \cdot 8 \\
1 \cdot 0 \\
1 \cdot 1 \\
1 \cdot 2\end{array}$ \\
\hline $\begin{array}{l}\text { Mean } \\
\pm \text { S.D. }\end{array}$ & $\begin{array}{l}0.5 \\
0.26\end{array}$ & $\begin{array}{l}1 \cdot 6 \\
1 \cdot 20\end{array}$ & $\begin{array}{l}1 \cdot 2 \\
0 \cdot 24\end{array}$ & $\begin{array}{l}1 \cdot 0 \\
0 \cdot 15\end{array}$ \\
\hline
\end{tabular}

${ }^{a}$ Wilcoxon test for pair differences. n.s. = not significant.

TABLE II Results in leucocyte migration and lymphocyte transformation tests when stimulating lymphocytes from patients with primary and secondary syphilis with T. pallidum antigen before, 1 month, and 2 to 6 months after treatment. Results expressed as migration indices (MI) and stimulation indices (SI)

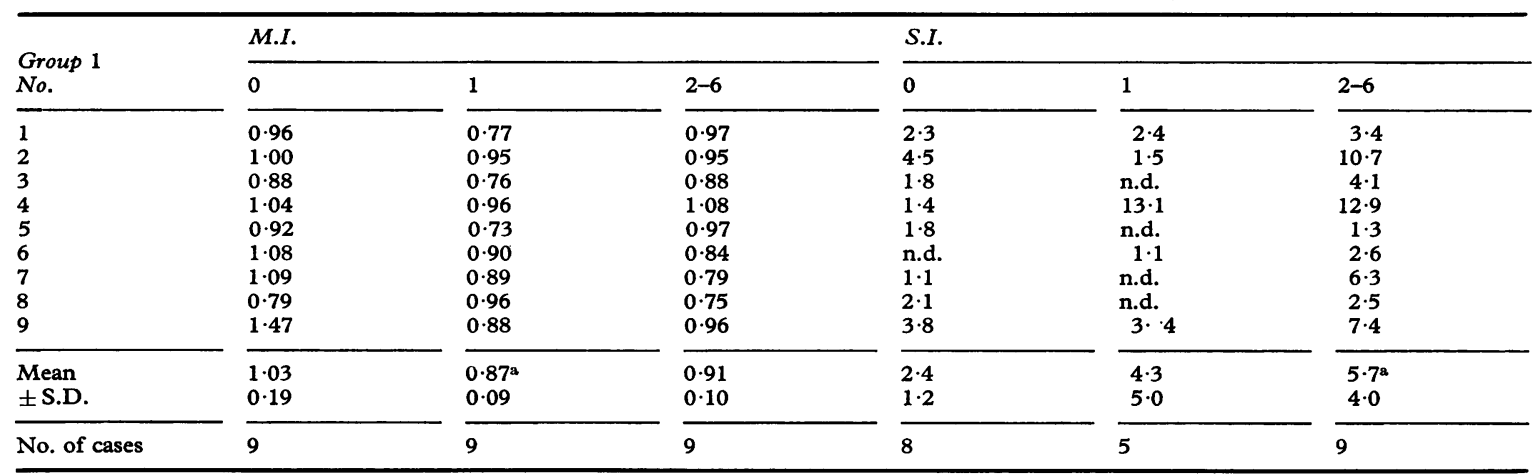

aP $<0.05$. Wilcoxon test for pair differences. n.d. $=$ not done. 


\section{Leucocyte migration test}

The response to TP-antigen in Group $\mathrm{I}$ is given in Table II. With one exception the migration index was lower 1 month after treatment than before treatment. The mean difference was found to be significant $(P<0.05)$. The mean migration index before treatment was $1.03,1$ month after treatment $0 \cdot 87$, and 2-6 months after treatment 0.91 .

Fig. 1 shows the areas from control cultures for each patient. No antigen was added and the leucocytes were allowed to grow out spontaneously into the agar gel. One month after treatment all the areas but one were greater than before treatment, and the difference was significant $(P<0 \cdot 05)$. 2-6 months after treatment the areas were significantly greater than the pre-treatment areas $(P<0.02)$.

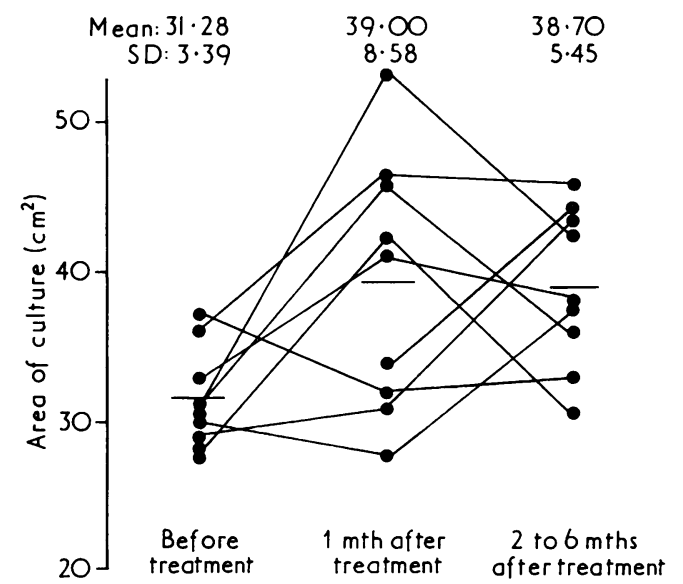

FIG. 1 Areas of control cultures of leucocytes from patients with primary and secondary syphilis before and after treatment. Results indicated in $\mathrm{cm}^{2}$.

No specific inhibition could be demonstrated in the mean migration indices from Group II and Group III compared with the control group (Table III).
Lymphocyte transformation test

The results after in vitro stimulation with TP-antigen in a LTT is shown in Table II. Four of eight patients in Group I showed a positive response to stimulation with TP-antigen during the acute infection. Eight of nine patients had a S.I. $>2.0$ 2-6 months after their first investigation. This increase in reactivity in vitro towards $\mathrm{TP}$-antigen was significant $(\mathbf{P}<0.05$; Wilcoxon test of pair differences). The patient who remained negative had secondary syphilis and was also non-reactive to TP-antigen in the LTT at the first investigation.

Two of four patients who had been treated for syphilis earlier (Group II) gave a S.I. $\geqslant 2 \cdot 0$ and so did two of four in Group III (Table III).

In comparing the results from the LTT with those obtained in the LMT in patients in Group I, no correlation could be found, even though the M.I.s observed one month after treatment were significantly lower than those seen during the acute disease. In the control group three patients showed increased migration indices $(M I>1 \cdot 10)$. In two of these the S.I. was above $2 \cdot 0$.

The results after stimulation with PHA in vitro are given in Fig. 2. The range of the responses is large, and no significant difference was found between any of the groups and the control group. The lowest PHA response during the acute illness was found in the patient who continued to give a negative response to TP-antigen. The patient in Group II with a PHA-response equal to 6.500 was a man with parenchymatous neurosyphilis. $\mathrm{He}$ was negative in the LTT to TP-antigen stimulation, but had positive STS.

In six patients in Group I, two with primary and four with secondary syphilis, plasma samples taken before, 1 month, and 2 to 6 months after treatment were investigated for immunosuppressive factors (Table IV). When normal lymphocytes were stimulated with PHA in patients' plasma there was no

TABLE III Results in leucocyte migration and lymphocyte transformation tests when stimulating lymphocytes from patients treated for syphilis 1 to 4 years earlier (Group 2), from patients with a reactive TPI but without known exposure to syphilis (Group 3), and from a control group. Results expressed as migration indices (MI) and stimulation indices (SI)

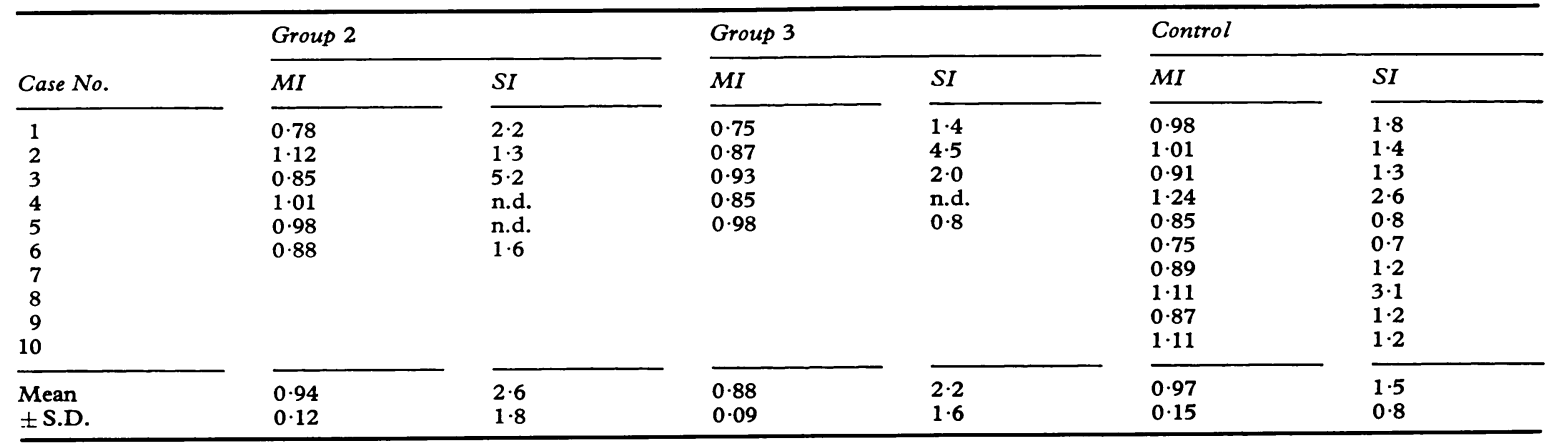




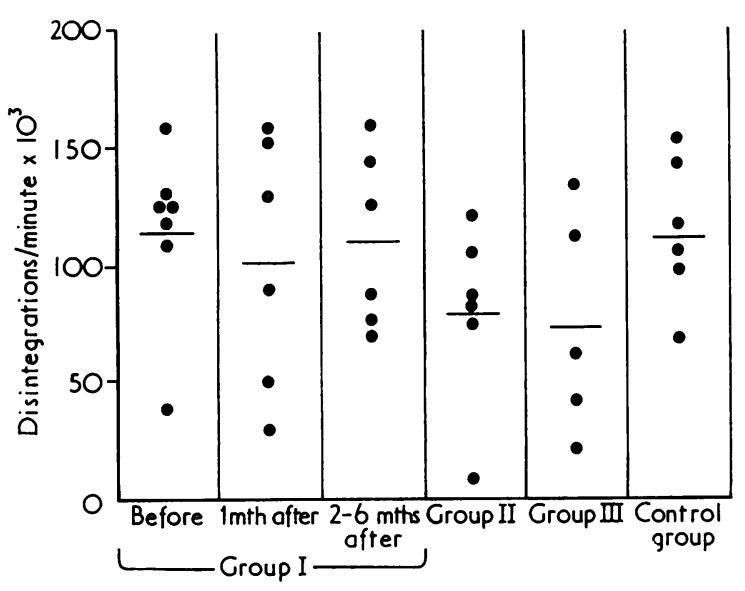

FIG. 2 Results of in vitro stimulation with PHA $1 \mathrm{\mu g} / \mathrm{ml}$ in patients with primary and secondary syphilis before, 1 month, and 2 to 6 months after treatment (Group 1), in patients treated for syphilis 1 to 4 years earlier (Group 2), in patients with a reactive TPI, but without known exposure to syphilis (Group 3), and in a control group. The results are expressed as disintegrations per minute $\times 10^{3}$.

TABLE IV Response of normal lymphocytes with $P H A$, Con $A$, and PWM in a lymphocyte transformation test after addition of patient's plasma taken before, 1 month, and 2 to 6 months after treatment. Reactivity of lymphocytes expressed as percentage of their reactivity when cultured in $A$-serum. See text.

\begin{tabular}{|c|c|c|c|}
\hline Months after treatment & $\begin{array}{l}\text { PHA } \\
\text { Per cent. }\end{array}$ & $\begin{array}{l}\text { CON A } \\
\text { Per cent. }\end{array}$ & $\begin{array}{l}P W M \\
\text { Per cent. }\end{array}$ \\
\hline $\begin{array}{l}0 \\
1 \\
2-6\end{array}$ & $\begin{array}{l}113 \\
102 \\
102\end{array}$ & $\begin{array}{c}75 \\
72 \\
103^{\mathrm{a}}\end{array}$ & $\begin{array}{c}114 \\
98 \\
93^{b}\end{array}$ \\
\hline
\end{tabular}

aP $<0.05$ paired $T$-test

bP $<0.01$ paired $T$-test

significant difference between the mean response after the addition of plasma taken before and after treatment. However, when the lymphocytes were stimulated with Con $\mathrm{A}$, there was a significant difference in reactivity, in that plasma taken during the disease and one month after treatment induced a decreased response $(P<0.05)$. This was reversed when the lymphocytes were stimulated with poke-weed mitogen $(P<0.01)$.

When the lymphocytes from three patients with secondary syphilis were cultured in 20 per cent. plasma without A-serum, there was a decreased reaction after the addition of suboptimal doses of PHA in plasma taken from two patients during their illness. The reactivity of the lymphocytes to suboptimal Con A concentrations was lowered after addition of both plasma samples, whereas the reactivity after PWM stimulation showed no clear change in the results.

\section{Discussion}

Previous investigations in vitro and in vivo indicate a suppressed or decreased cell-mediated immune reactivity in early infectious syphilis (Festenstein, Abrahams, and Bokkenheuser, 1967; Levene, Wright, and Turk, 1971; Musher, Schell, and Knox, 1974; Musher, Schell, Jones, and Jones, 1975; Wright and Grimble, 1974). Our results support these data. Moreover, in our study, both the LMT and the LTT show significantly increased reactivity in vitro after treatment, although only briefly in the LMT. This confirms preliminary results of Friedmann and Turk (1975). Similar changes in lymphocyte reactivity have been found in patients with oral candidiasis, where an LMT with Candida antigen became temporarily positive after treatment (Budtz-Jørgensen, 1973).

The low lymphocyte reactivity and the decrease in skin reactivity could be due to high amounts of antigen present in the patients. Clearing of antigen was followed by an increase in in vitro and in vivo parameters. This hypothesis is further supported by the observation that a tuberculin skin test can induce a temporary suppression of the reactivity in vitro of blood lymphocytes after stimulation with tuberculin (Thestrup-Pedersen, 1974) and that a primary skin reaction towards tuberculin reduces the size of a secondary tuberculin skin reaction taking place 2 days after the first test (Thestrup-Pedersen, 1975). This, however, does not explain why in vitro responses were depressed to unrelated antigens such as Candida and trichophytin (Musher and others, 1975) and PPD (Friedmann and Turk, 1975). The changed reactivity of the macrophages during the acute infection (Fig. 1) could explain at least the depressed skin reactions and maybe also the observations in vitro.

Another possible explanation for the decrease in the reactivity may be the presence of an inhibitory activity found in fresh plasma of patients with secondary syphilis (Levene, Wright, Turk, and Grimble, 1969). However, this inhibition of PHA reactivity of lymphocytes was not found in serum by Musher and others (1974) or in our experiments using stored syphilitic plasma (Table IV). The response to Con $A$ stimulation was suppressed and plasma from syphilis patients seems therefore to be able to change the in vitro activity of $T$ cells in certain respects. A more extensive investigation has been performed on the effect of plasma from 81 syphilis patients on normal lymphocytes (Thompson, 
Dwyer, Mangi, and Lee, unpublished observations). This suggested that some patients with secondary syphilis had plasma factors which significantly suppressed the reactivity of normal lymphocytes to PHA stimulation. These authors indicate that such inhibitory factors may be markers of suppressor $T$ cells. The results reported so far could support this hypothesis of a non-specific suppressor mechanism which mainly affects $T$ cell functions and leaves the B cells capable of producing various antibodies. It may be more than a coincidence that the reactivity of both $T$ and $B$ cells (PWM stimulation) was increased after the addition of plasma to the cultures (Table IV). This observation needs further investigation.

This study was supported by grant number $512-4200$ from the Danish Medical Research Council and by P. Carl Petersens Foundation. The technical assistance by Mrs. Anne Knudsen, Mrs. Karen Skibsted and Mrs. Vibeke Thulin is gratefully acknowledged.

We also acknowledge the supply of Treponema pallidum antigen by Treponematoseafdelingen, Statens Seruminstitut, Copenhagen, and we thank the Statens Seruminstitut for undertaking the serum antibody tests.

\section{References}

Budtz-Jørgensen, E. (1973) Scand. F. dent. Res., 81, 372 Böyum, A. (1968) Scand. F. clin. Lab. Invest., 21, Suppl. 97, p.9

Clausen, J. E. (1973) Acta allerg. (Kbh.), 28, 351

Festenstein, H., Abrahams, C., and Bokkenheuser, V. (1967) Clin. exp. Immunol., 2, 311

FriedmanN, P. S., and TURK, J. L. (1975) Ibid., 21, 59

Fulford, K. W. M., and BrostofF, J. (1972) Brit. f, vener. Dis., 48, 483

LEVENE, G. M., WRIGHT, D. J. M., and TURK, J. L. (1971) Proc. roy. Soc. Med., 64, 426

2, $\frac{246}{-}-$, and GrimbLe, A. G. S. (1969) Lancet,

MANGi, R. J., DwYeR, J. M., and Kantor, F. S. (1974) Clin. exp. Immunol., 18, 519

MuShER, D. M., SChell, R. F., JoNes, R. F., and JoNes, A. M. (1975) Infect. and Immun., 11, 1261

$\longrightarrow$, , and KNOX, J. M. (1974) Ibid., 9, 654

Thestrup-Pedersen, K. (1974) Immunology, 27, 965

- (1975) Ibid., 28, 343

Thulin, H., Thestrup-Pedersen, $\mathrm{K}$., and Ellegaard, J. (1975) Acta allerg. 'Kbh.), 30, 9

WRIGHT, D. J. M., and Grimble, A. S. (1974) Brit. f. vener. Dis., 50, 45 